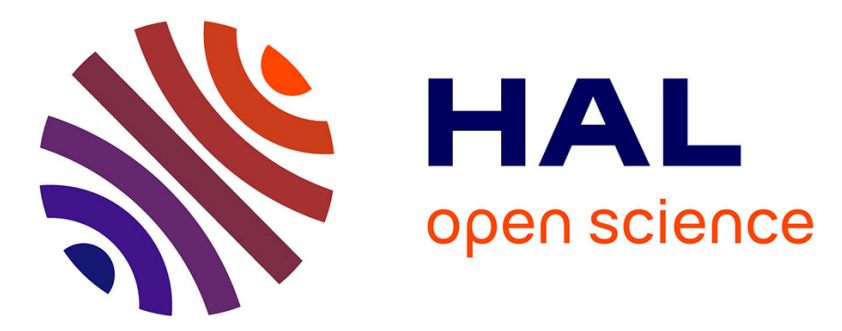

\title{
A dedicated state space for power system modeling and frequency and unbalance estimation
}

\author{
Tuan Anh Phan, P. Wira, Gilles Hermann
}

\section{To cite this version:}

Tuan Anh Phan, P. Wira, Gilles Hermann. A dedicated state space for power system modeling and frequency and unbalance estimation. Evolving Systems, 2017, 9 (1), pp.57-69. 10.1007/s12530-0179177-9 . hal-02306227

\section{HAL Id: hal-02306227 https://hal.science/hal-02306227}

Submitted on 4 Oct 2019

HAL is a multi-disciplinary open access archive for the deposit and dissemination of scientific research documents, whether they are published or not. The documents may come from teaching and research institutions in France or abroad, or from public or private research centers.
L'archive ouverte pluridisciplinaire HAL, est destinée au dépôt et à la diffusion de documents scientifiques de niveau recherche, publiés ou non, émanant des établissements d'enseignement et de recherche français ou étrangers, des laboratoires publics ou privés. 


\title{
A Dedicated State Space for Power System Modeling and Frequency and Unbalance Estimation
}

\author{
Anh Tuan Phan, Patrice Wira, Gilles Hermann
}

\begin{abstract}
Over the last decades, a great deal of research has been focused on power quality issues in electrical energy transportation. We present a statespace representation to model dynamical power systems like electrical distribution systems. The proposed model is able to take into account all the dynamic behavior of a multiphase power system. It has been applied to model a typical three-phase power system and its unbalance, i.e., an electrical grid which can be perturbed by nonlinear loads and distributed renewable energy generation which is a typical changing system. Associated with an extended Kalman filter, the state-space model is used to iteratively estimate power quality parameters. Indeed, the symmetrical components of the power system, i.e., their amplitude and phase angle values, and the fundamental frequency can be calculated at each iteration without any prior knowledge. The proposed estimation technique is an evolving and adaptive method able to handle the changing power system. Its effectiveness has been evaluated by several tests. Results have been compared to other methods. They show the efficiency and better performance of the proposed method. The fundamental frequency and the symmetrical components are precisely estimated even under disturbed and time-varying conditions. This state-space representation can therefore be used in active power filtering schemes and in load frequency control strategies.
\end{abstract}

Keywords State-Space Model · Power Systems · Power Quality · Parameter Estimation · Unbalance · Symmetrical Components · Fundamental Frequency Tracking · Extend Kalman Filter

Anh Tuan Phan, Patrice Wira, Gilles Hermann

Laboratoire Modélisation, Intelligence, Processus et Systèmes (MIPS - EA 2332)

Université de Haute Alsace

61 rue Albert Camus, 68093 Mulhouse Cedex France

E-mail: anh-tuan.phan@uha.fr, patrice.wira@uha.fr, gilles.hermann@uha.fr

Cite this article as:

Phan, A. T., Wira, P., \& Hermann, G. (2018). A dedicated state space for power system modeling and frequency and unbalance estimation. Evolving Systems, 9(1), 57-69.

https://doi.org/10.1007/s12530-017-9177-9 


\section{Introduction}

In ideal power distribution systems, an electrical signal like a voltage or a current is a single, constant-frequency sinusoid. However and in practice, electrical signals are disturbed and distorted. Indeed, electrical signals are influenced by noise and composed of the sum of harmonically related sinusoids, i.e., the fundamental-frequency term and higher-order frequency terms called harmonics [2]. The frequency values of the harmonics are multiples of the fundamental frequency. The main frequency value rarely stays constant; some devices can generate reactive power and cause unbalanced voltage situations in the case of multiphase systems [12]. The electric utilities are expected to provide continuous and quality power to the customers, i.e., with specified voltage and frequency limits. This is referred to as power quality [3], [10]. Mathematically, power quality can be defined with a set of several parameters and indicators, obviously, some of them have a physical interpretation [9]. Estimating and monitoring these parameters in real-time allows to prevent against troubles and failures [15] ; actions can thus be operated in an appropriate way [22], [32].

The fundamental frequency is a key parameters of a power system. In realworld applications, the frequency value varies according to the power demand. Indeed, the fundamental frequency rises if the power generation is higher than the power demand and falls otherwise. The power generation and demand vary continuously and lead to small fundamental frequency variations.

A three phase power system is balanced if its three phase currents (and three phase voltages) are equal in magnitudes and phase-shifted by $120^{\circ}$ from each other; if not, the system is unbalanced. Unbalance conditions come from the uneven distribution of single-phase loads, asymmetrical transformer winding impedances, asymmetrical transmission impedances, unbalanced and overloaded equipment [16]. Sometimes, they can be the consequence of asymmetrical faults such as line to ground, two lines to ground, or line to line connections. The unbalance of a power system causes power loss, heating, bad performance of induction machines and power electronic converters and drives [16]. Theory of symmetrical components is a powerful tool to analyze unbalance conditions [4], therefore identifying the symmetrical components plays an important rule in improving the reliability and stability of power systems.

The objective of this work is to model the three phase signals of a power system even under unbalanced conditions with an original state-space representation. A new state-space is therefore developed and an Extended Kalman Filter (EKF) is associated for estimating the states in real-time. Therefore, the proposed model yields to estimate the fundamental frequency and to identify the symmetrical components of the power system, i.e., their amplitudes and phase angles. The method can be inserted in an Active Power Filtering (APF) scheme for real-time operations as represented by Fig. 1. This means that the estimated parameters can be used in power quality enhancement strategies.

The organization of this paper is as follows. In Sect. 2, important issues in power quality, frequency estimation and symmetrical components are highlighted. In Sect. 3, a new state-space model able to model the unbalance of a 


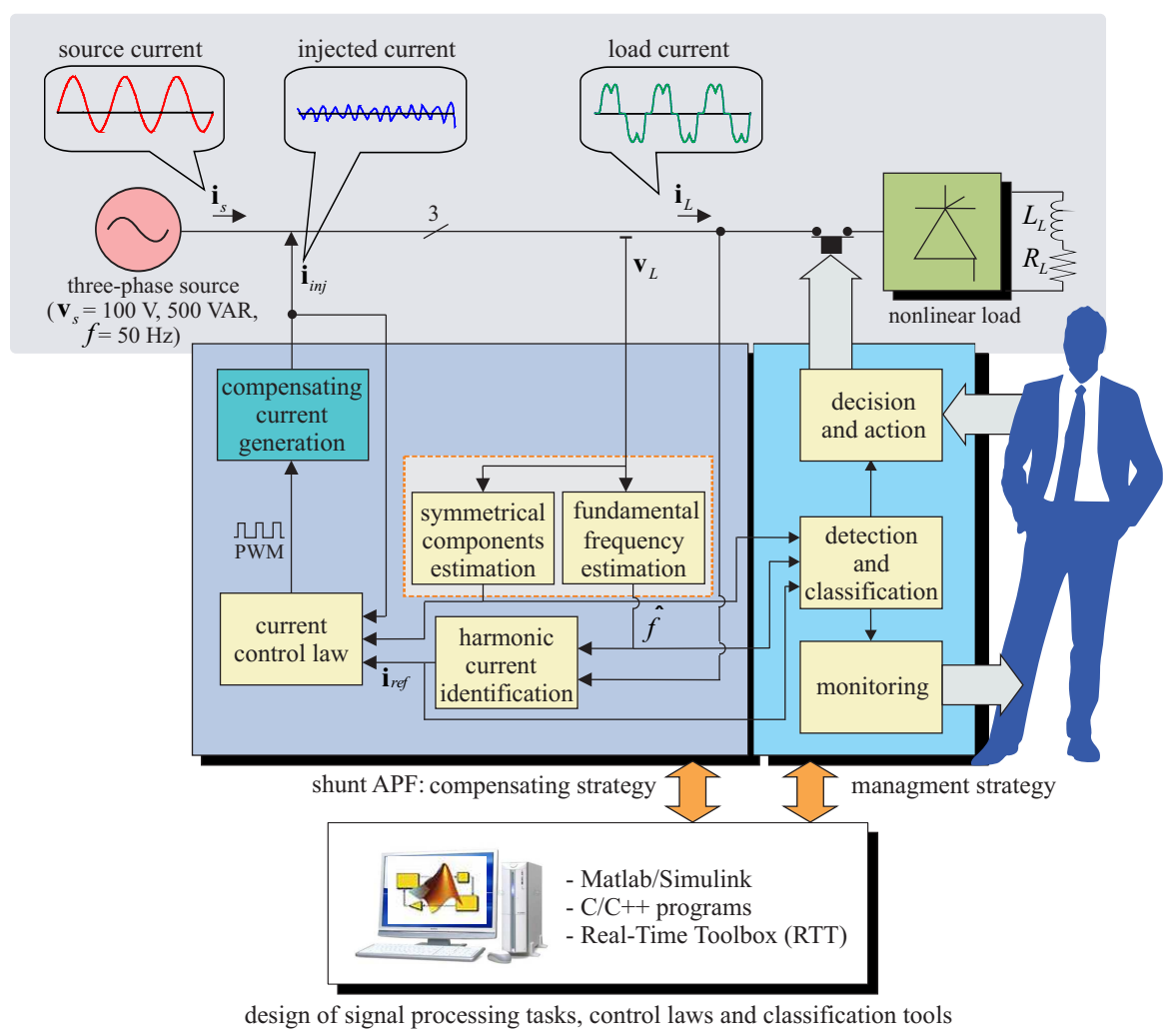

Fig. 1 Operating principles of a shunt active power filter for power quality enhancement

power system is introduced. The proposed state-space associated to a Kalman filter implementation is proposed in Sect. 4 in order to estimate the fundamental frequency and the symmetrical components. The performance of the proposed method is evaluated for frequency estimation in Sect. 5 and for symmetrical components estimation in Sect. 6. Some concluding remarks are stated in Sect. 7.

\section{Power quality parameters}

\subsection{System's parameters}

Grid voltage conditions such as phase, amplitude and frequency determine the proper operation of a grid connected system. In such applications, a fast and accurate detection of the phase angle, frequency and amplitude of the grid voltage is essential. These factors, together with the implementation simplicity and the cost are all important when examining the credibility of monitoring and compensation strategy. Therefore an ideal parameter estimation scheme must be used to promptly and smoothly track the grid behavior through vari- 
ous short-term disturbances and long term disturbances and to set the energy transfer between the grid and the power converter. The energy must also be shared properly into the phases of multiphase systems.

The power generation and demand vary continuously. A part of the energy generation is difficult to be controlled or even to be predicted, i.e., energy from renewable sources. On the other side, energy absorbed by loads depends on the users through domestic or industrial facilities. A power distribution system can thus be considered as a non-stationary process and Fourier's theory can therefore not be used.

The three voltages of a discrete three-phase power system can be expressed by the following general formulation:

$$
\left\{\begin{array}{l}
v_{a}(k)=V_{a} \sin \left(\omega k T_{s}+\phi_{a}\right) \\
v_{b}(k)=V_{b} \sin \left(\omega k T_{s}+\phi_{b}\right) \\
v_{c}(k)=V_{c} \sin \left(\omega k T_{s}+\phi_{c}\right)
\end{array}\right.
$$

where $\omega$ is the angular speed, $k$ is the iteration number and $T_{s}$ stands for the sampling period. This system can be either balanced or unbalanced. It can be seen that the amplitudes $V_{a}, V_{b}$ and $V_{c}$ are not necessary equal and that the phase angles $\phi_{a}, \phi_{b}$ and $\phi_{c}$ are not necessary shifted from $\frac{2 \pi}{3}$ each other. Additionally, the frequency reference of this system is given by $\omega=2 \pi f$ with $f$ the fundamental frequency subject to variations.

\subsection{Fundamental frequency estimation}

Online fundamental frequency estimation, and moreover power systems' parameters identification can be achieved according to various methods and models. The simple zero-crossing technique [21] however is sensitive to noise and harmonics. Robust Phase-Locked Loop (PLL) methods have been developed [33], [20], but they need a time-delay. Fourier's theory and Discrete Fourier Transforms (DFT) have been used like in [35], however they suppose the signals to be stationary. Beside frequency-domain methods, some timedomain methods are also good candidates. Some are reviewed thereafter. Each different method assumes different signal models but should be able to cope with real-time demands, i.e., changing operation conditions, varying environmental influences, and drifts. Indeed, they have to be adaptive by tacking into account the latest measured signals from the electric grid, i.e., the voltages and currents. Furthermore, they have to be compliant with severe environments: The presence of noise, higher-order harmonics, unbalanced conditions.

For example, the adaptive Prony's method [27] tries to model a single phase voltage/current as a sum of exponential functions in order to estimate the frequencies, amplitudes and phases of the fundamental and harmonic components. State-space models have been designed for estimating the fundamental frequency iteratively. The state-space is build from a single phase voltage in [15], three phase voltages are taken into account in [7]. However, all these 
models only deal with either a single phase of a power system (as the ones in [27], [15]) or a balanced three phase power system (as in [7]).

Least Mean Square (LMS) algorithms which exploit the harmonic structure of the sinusoidal signal have been successfully developed in [26]. In [6], the MUSIC approach has been applied, based on an initial estimate and an optimally weighted least squares cost function. Time-frequency methods are able to estimate the fundamental frequency under some conditions [5]. Another time-domain based power system frequency estimation algorithm has been proposed in [31]. This approach relies on the discrete forms of voltage signal and a wavelet transform has been implemented for de-noising the signal before processing. The wavelet transform has the advantage over the Fourier transform that is the ability to maintain time-domain information of the signals. Using a variable window size, wavelets are able to detect both high and low frequency contents of signals. Beside, the Kalman Filter (KF) is known to be an efficient tool for estimating and tracking frequencies in power systems [7], [23]. Of course, KF schemes rely on an appropriate state-space model, i.e., that is able to precisely reproduce the behavior of the considered system. The extended KF has been recently modified in [30], [28], [29], [11] for the online learning of nonlinear systems. A KF with a linear quadratic regulator is proposed in [18] to improve the power quality by taking into account grid perturbations such as load variations, frequency deviation, voltage distortion, line impedance, unbalance, and measurement noise.

Non-parametric methods can also be considered for estimating the parameters of a power systems. Specific neural approaches have shown good capabilities in estimating the fundamental frequency of electrical systems [8], [13]. Other data-driven approach or even evolving auto-regressive models can be used [17], [34]. Their potential in signal recovering are sometimes counterbalanced by their computational costs.

\subsection{Symmetrical components for unbalance modeling}

The system in Eq. 1 can always be expressed as an unique sum of symmetrical components [1], [4]:

$$
\begin{aligned}
& {\left[\begin{array}{c}
v_{a}(k) \\
v_{b}(k) \\
v_{c}(k)
\end{array}\right]=\left[\begin{array}{c}
V_{+} \sin \left(\omega k T_{s}+\phi_{+}\right) \\
V_{+} \sin \left(\omega k T_{s}+\phi_{+}-\frac{2 \pi}{3}\right) \\
V_{+} \sin \left(\omega k T_{s}+\phi_{+}+\frac{2 \pi}{3}\right)
\end{array}\right]+} \\
& \left.\begin{array}{c}
V_{-} \sin \left(\omega k T_{s}+\phi_{-}\right) \\
V_{-} \sin \left(\omega k T_{s}+\phi_{-}+\frac{2 \pi}{3}\right) \\
V_{-} \sin \left(\omega k T_{s}+\phi_{-}-\frac{2 \pi}{3}\right)
\end{array}\right]+\left[\begin{array}{c}
V_{o} \sin \left(\omega k T_{s}+\phi_{o}\right) \\
V_{o} \sin \left(\omega k T_{s}+\phi_{o}\right) \\
V_{o} \sin \left(\omega k T_{s}+\phi_{o}\right)
\end{array}\right] .
\end{aligned}
$$

In the previous expression, the positive components are written with subscripts + , the negative components with - and the zero components with o. These components are defined by their amplitudes and their phase angles. The system in Eq. 1 is balanced when $V_{-}=V_{o}=0$ (this configuration represents 
a three-phase, balanced, positive sequence system) or when $V_{+}=V_{o}=0$ (this represents a three-phase, balanced, negative sequence system).

Any three-phase voltages can be converted into the two-dimensional $\alpha \beta$ reference frame with the Clarke transformation by using:

$$
C=\sqrt{\frac{2}{3}}\left[\begin{array}{cc}
1 & 0 \\
-\frac{1}{2} & \frac{\sqrt{3}}{2} \\
-\frac{1}{2} & -\frac{\sqrt{3}}{2}
\end{array}\right] .
$$

The positive, negative and zero components can be converted in the $\alpha \beta$ reference frame:

$$
\begin{gathered}
{\left[\begin{array}{c}
v_{\alpha}^{+} \\
v_{\beta}^{+}
\end{array}\right]=C^{T}\left[\begin{array}{c}
V_{+} \sin \left(\omega k T_{s}+\phi_{+}\right) \\
V_{+} \sin \left(\omega k T_{s}+\phi_{+}-\frac{2 \pi}{3}\right) \\
V_{+} \sin \left(\omega k T_{s}+\phi_{+}+\frac{2 \pi}{3}\right)
\end{array}\right],} \\
{\left[\begin{array}{c}
v_{\alpha}^{-} \\
v_{\beta}^{-}
\end{array}\right]=C^{T}\left[\begin{array}{c}
V_{-} \sin \left(\omega k T_{s}+\phi_{-}\right) \\
V_{-} \sin \left(\omega k T_{s}+\phi_{-}+\frac{2 \pi}{3}\right) \\
V_{-} \sin \left(\omega k T_{s}+\phi_{-}-\frac{2 \pi}{3}\right)
\end{array}\right],} \\
{\left[\begin{array}{c}
v_{\alpha}^{o} \\
v_{\beta}^{o}
\end{array}\right]=0 .}
\end{gathered}
$$

As it can be seen, the zero component in the $\alpha \beta$-reference frame is eliminated.

The corresponding complex expressions of the positive and negative components are respectively calculated from Eq. 4 and Eq. 5 and yield to:

$$
\begin{gathered}
v^{+}(k)=v_{\alpha}^{+}(k)+j v_{\beta}^{+}(k)=A_{+} e^{j \omega k T_{s}} . \\
v^{-}(k)=v_{\alpha}^{-}(k)+j v_{\beta}^{-}(k)=A_{-} e^{-j \omega k T_{s}} .
\end{gathered}
$$

With the Clarke transformation, the resulting complex expression of the three phase signals is the sum of the complex voltages corresponding to the positive, negative and zero components and is:

$$
v(k)=A_{+} e^{j \omega k T_{s}}+A_{-} e^{-j \omega k T_{s}} .
$$

\section{A state-space for modeling unbalanced power systems}

\subsection{Proposed state-space}

An original state-space model is developed thereafter. This model does not require the fundamental frequency value and is able to represent any threephase power system in both balanced and unbalanced conditions. This is not the case of lots of existing models. The advantage of the proposed state-space representation is that it gives directly access to some physical parameters of the system, like the value of the fluctuating fundamental frequency [25] and the amplitude and phase values of the voltage symmetrical components [24]. 
The three following states are chosen to define the state-space corresponding to a three-phase power system:

$$
\left\{\begin{array}{l}
q_{1}(k)=e^{j \omega k T_{s}}=\cos \left(\omega k T_{s}\right)+j \sin \left(\omega k T_{s}\right) \\
q_{2}(k)=A_{+} e^{j \omega k T_{s}} \\
q_{3}(k)=A_{-} e^{-j \omega k T_{s}}
\end{array}\right.
$$

Variables $q_{1}(k), q_{2}(k)$ and $q_{3}(k)$ stands for the states of the system; they have a physical interpretation. Thus, $q_{2}(k)$ and $q_{3}(k)$ respectively represent the positive and negative components of the three phase system given by Eq. 1 . In addition, $q_{1}(k)$ represents the variation of the phase angle of $q_{2}(k)$ and $q_{3}(k)$ between two consecutive iterations. The consequence is that these three states can model not only balanced but also unbalanced three phase systems.

By writing $q_{1}(k+1), q_{2}(k+1)$ and $q_{3}(k+1)$ as functions of $q_{1}(k), q_{2}(k)$ and $q_{3}(k)$, the states can be expressed in an iterative way leading to the following state-space model with a scalar output:

$$
\begin{gathered}
{\left[\begin{array}{l}
q_{1}(k+1) \\
q_{2}(k+1) \\
q_{3}(k+1)
\end{array}\right]=\left[\begin{array}{ccc}
1 & 0 & 0 \\
0 & q_{1}(k) & 0 \\
0 & 0 & \frac{1}{q_{1}(k)}
\end{array}\right]\left[\begin{array}{l}
q_{1}(k) \\
q_{2}(k) \\
q_{3}(k)
\end{array}\right],} \\
y(k)=\left[\begin{array}{lll}
0 & 1 & 1
\end{array}\right]\left[\begin{array}{lll}
q_{1}(k) & q_{2}(k) & q_{3}(k)
\end{array}\right]^{T} .
\end{gathered}
$$

The model proposed by Eq. 11 is able to describe any general three-phase power system, i.e., balanced or unbalanced. The implicit assumption about this model is that choosing $e^{j \omega k T_{s}}$ as a state remains to consider its evolution as linear between two time samples [24].

Obviously, it can be inserted in an iterative identification scheme in order to estimate in real-time the states and the parameters of any evolving power system.

\subsection{Utilization of the proposed state-space with an EKF scheme}

The proposed state-space given by Eq. 11 is nonlinear and can be used in the implementation of an EKF scheme. The state vector to be estimated is $\mathbf{q}(k)=\left[q_{1}(k) q_{2}(k) q_{3}(k)\right]^{T} ;$ Eq. 11 and Eq. 12 can respectively be rewritten with the following general expressions:

$$
\begin{gathered}
\mathbf{q}(k+1)=\mathbf{F}(\mathbf{k}, \mathbf{q}(\mathbf{k}))+\nu_{\mathbf{1}}(\mathbf{k}), \\
\mathbf{y}(\mathbf{k})=\mathbf{C}(\mathbf{k}, \mathbf{q}(\mathbf{k}))+\nu_{\mathbf{2}}(\mathbf{k}),
\end{gathered}
$$

where $\boldsymbol{\nu}_{\mathbf{1}}(k)$ and $\boldsymbol{\nu}_{\mathbf{2}}(k)$ are uncorrelated, zero-mean, white noises. The output vector $\mathbf{y}(k)$ is supposed to be known or measured from the system. The functions $\mathbf{F}()$ or $\mathbf{C}()$ are non-linear functions that represent the dynamics of the system whose states have to be estimated. The EKF is used to estimate the state vector by minimizing the Mean Squared Error (MSE) based on the real 
values of the state vector. This error is updated iteratively by error-correction for estimating the states [19].

The recursive equations of the discrete EKF are recapitulated [14]:

$$
\begin{gathered}
\mathbf{G}_{\mathbf{f}}(k)=\mathbf{K}(k, k-1) \mathbf{C}^{H}(k) \times \\
{\left[\mathbf{C}(k) \mathbf{K}(k, k-1) \mathbf{C}^{H}(k)+\mathbf{Q}_{\mathbf{2}}(k)\right]^{-1}} \\
\boldsymbol{\alpha}(k)=\mathbf{y}(k)-\mathbf{C}\left(k, \widehat{\mathbf{q}}\left(k \mid \mathbf{Y}_{k-1}\right)\right) \\
\widehat{\mathbf{q}}\left(k \mid \mathbf{Y}_{k}\right)=\widehat{\mathbf{q}}\left(k \mid \mathbf{Y}_{k-1}\right)+\mathbf{G}_{\mathbf{f}}(k) \boldsymbol{\alpha}(k) \\
\widehat{\mathbf{q}}\left(k+1 \mid \mathbf{Y}_{k}\right)=\mathbf{F}\left(k, \widehat{\mathbf{q}}\left(k \mid \mathbf{Y}_{k}\right)\right) \\
\mathbf{K}(k)=\left[\mathbf{I}-\mathbf{G}_{\mathbf{f}}(k) \mathbf{C}(k) \mathbf{K}(k, k-1)\right] \\
\mathbf{K}(k+1, k)=\mathbf{F}(k+1, k) \mathbf{K}(k) \mathbf{F}^{H}(k+1, k)+\mathbf{Q}_{\mathbf{1}}(k) \\
\mathbf{F}(k+1, k)=\frac{\partial \mathbf{F}(k, \mathbf{q})}{\partial \mathbf{q}} \mid \widehat{\mathbf{q}}\left(k \mid \mathbf{Y}_{k}\right), \\
\mathbf{C}(k)=\frac{\partial \mathbf{C}(k, \mathbf{q})}{\partial \mathbf{q}} \mid \widehat{\mathbf{q}}\left(k \mid \mathbf{Y}_{k-1}\right) .
\end{gathered}
$$

In Eq. 15 to Eq. 22, the conjugate transpose of a matrix is marked with the letter $\mathrm{H}$ in exponent. Additionally, $\widehat{\mathbf{q}}\left(k \mid \mathbf{Y}_{k}\right)$ is the estimated state vector at iteration $k, \mathbf{Q}_{\mathbf{1}}(k)$ is the correlation matrix of the process noise vector $\nu_{1}(k)$, $\mathbf{Q}_{\mathbf{2}}(k)$ is the correlation matrix of the measurement noise vector $\nu_{2}(k)$ and I stands for the identity matrix. Additionally, $\mathbf{Y}(k)$ means the observation set, i.e., all the measurements from the first to the actual iteration: $\mathbf{Y}(k)=$ $[\mathbf{y}(1), \mathbf{y}(2), \ldots, \mathbf{y}(k)]$. It is included in the previous equations to express the fact that some parameters depend on all the past measure values, i.e., takes into account the dynamics of the system. $\mathbf{F}()$ in Eq. 13 denotes a nonlinear transition matrix function that is time-variant and that depends on one state. This non-linearity is simplified by a linearized model given by Eq. 21 at each iteration.

The implementation of the discrete EKF consists in updating Eq. 15 to Eq. 22, on each iteration, i.e., for $k=1,2,3, \ldots$. Initial conditions have to be set, i.e., the predicted state error correlation matrix and the predicted state: $\mathbf{K}(1,0)$ and $\widehat{\mathbf{q}}(1 \mid \mathbf{Y}(0))$. One should note that using KF or EKF schemes needs some assumptions: $\nu_{1}(k)$ and $\nu_{2}(k)$ are white processes that are uncorrelated with $\mathbf{q}(k)$ and with each other; $\mathbf{Q}_{\mathbf{2}}(k)>0$.

In our application, the nonlinear state model in Eq. 13 corresponds to the proposed state-space of the three phase signals, i.e., Eq. 11. The approximate state-space is thus a linear model of Eq. 11 and Eq. 21 writes:

$$
\mathbf{F}(k, \mathbf{q}(k))=\left[\begin{array}{c}
q_{1}(k) \\
q_{1}(k) q_{2}(k) \\
\frac{q_{3}(k)}{q_{1}(k)}
\end{array}\right],
$$

Eq. 14 corresponds to Eq. 12, Eq. 22 thus writes:

$$
\mathbf{C}(k, \mathbf{q}(k))=\left[q_{2}(k)+q_{3}(k)\right] .
$$




\section{Power system parameters estimation with Kalman filtering under balanced or unbalanced conditions}

Power system states and parameters can be iteratively estimated by an EKF scheme relying on the proposed state-space model. This model is compliant to balanced and unbalanced three-phase power systems. The proposed estimator can be applied to stationary and non-stationary power systems with an unknown fundamental frequency. It can be noticed that no assumption have been made on the system's parameters and on their evolution, except the ones related to the Kalman filter. With a third-order state-space, the computational requirements of this estimator are limited, i.e., suitable for a real-time implementation that allows to monitor power system evolutions.

The following shows how the fundamental frequency and the symmetrical components can be deduced from $q_{1}(k), q_{2}(k), q_{3}(k)$, i.e., the states of the power system estimated at each iteration, by the proposed method.

\subsection{Estimation of the fundamental frequency}

As $q_{1}(k)$ represents the variation between two consecutive iterations of the phase angle of $q_{2}(k)$ and $q_{3}(k)$, the fundamental frequency of the power system can be directly estimated from the imaginary part of $q_{1}(k)$ according to:

$$
\hat{f}(k)=\frac{1}{2 \pi T_{s}} \sin ^{-1}\left(\operatorname{img}\left(q_{1}(k)\right)\right) .
$$

\subsection{Estimation of the symmetrical components}

The symmetrical components, i.e., the positive, negative and zero components of a three-phase signal are closely related to the state variables (according to Eq. 10). It is thus possible to deduce the three-phase components iteratively while running the EKF. This can be achieved according to the following [24].

The positive component is represented by $q_{2}(k)$, thus, its amplitude and phase angle can be estimated at each iteration by:

$$
\left\{\begin{array}{c}
V_{+}(k)=\sqrt{\frac{2}{3}} \overline{q_{2}}(k) \\
\phi_{+}(k)=\pi / 2+\angle q_{2}(k)-k \angle q_{1}(k)
\end{array},\right.
$$

where $\overline{q_{2}}(k)$ is the module of $q_{2}(k)$ and $\angle q_{1}(k)$ and $\angle q_{2}(k)$ are the phase angles of respectively $q_{1}(k)$ and $q_{2}(k): \angle q_{2}(k)=\omega k T_{s}+\phi_{+}(k)-\pi / 2$ and $\angle q_{1}(k)=\omega T_{s}$.

If $\left[v_{a}^{+}(k) v_{b}^{+}(k) v_{c}^{+}(k)\right]$ are the three phases of the positive component, then they can be reconstructed by applying the inverse $\alpha \beta$-transform:

$$
\left[\begin{array}{l}
v_{a}^{+}(k) \\
v_{b}^{+}(k) \\
v_{c}^{+}(k)
\end{array}\right]=\sqrt{\frac{2}{3}}\left[\begin{array}{cc}
1 & 0 \\
-\frac{1}{2} & \frac{\sqrt{3}}{2} \\
-\frac{1}{2} & -\frac{\sqrt{3}}{2}
\end{array}\right]\left[\begin{array}{l}
v_{\alpha}^{+}(k) \\
v_{\beta}^{+}(k)
\end{array}\right]=\sqrt{\frac{2}{3}}\left[\begin{array}{cc}
1 & 0 \\
-\frac{1}{2} & \frac{\sqrt{3}}{2} \\
-\frac{1}{2} & -\frac{\sqrt{3}}{2}
\end{array}\right]\left[\begin{array}{l}
\operatorname{Re}\left(q_{2}(k)\right) \\
\operatorname{Im}\left(q_{2}(k)\right)
\end{array}\right]
$$


where operators $\operatorname{Re}(z)$ and $\operatorname{Im}(z)$ denotes respectively the real and the imaginary parts of $z$.

In the same way as for the positive component, the parameters of the negative component are determined from state $q_{3}(k)$ according to

$$
\left\{\begin{array}{c}
V_{-}(k)=\sqrt{\frac{2}{3}} \overline{q_{3}}(k) \\
\phi_{-}(k)=\pi / 2-\angle q_{3}(k)-k \angle q_{1}(k)
\end{array}\right.
$$

where $\angle q_{3}(k)=\pi / 2-\omega k T_{s}-\phi_{-}(k)$.

The corresponding three phases $\left[v_{a}^{-}(k) v_{b}^{-}(k) v_{c}^{-}(k)\right]$ can thus be reconstructed from:

$$
\left[\begin{array}{l}
v_{a}^{-}(k) \\
v_{b}^{-}(k) \\
v_{c}^{-}(k)
\end{array}\right]=\sqrt{\frac{2}{3}}\left[\begin{array}{cc}
1 & 0 \\
-\frac{1}{2} & \frac{\sqrt{3}}{2} \\
-\frac{1}{2} & -\frac{\sqrt{3}}{2}
\end{array}\right]\left[\begin{array}{c}
v_{\alpha}^{-}(k) \\
v_{\beta}^{-}(k)
\end{array}\right]=\sqrt{\frac{2}{3}}\left[\begin{array}{cc}
1 & 0 \\
-\frac{1}{2} & \frac{\sqrt{3}}{2} \\
-\frac{1}{2} & -\frac{\sqrt{3}}{2}
\end{array}\right]\left[\begin{array}{l}
\operatorname{Re}\left(q_{3}(k)\right) \\
\operatorname{Im}\left(q_{3}(k)\right)
\end{array}\right]
$$

At the end, the zero component is directly calculated from the three-phase signals measured $v_{a}(k), v_{b}(k), v_{c}(k)$ at each iteration by:

$$
v_{a}^{o}(k)=v_{b}^{o}(k)=v_{c}^{o}(k)=\frac{v_{a}(k)+v_{b}(k)+v_{c}(k)}{3} .
$$

\section{Frequency estimation under unbalanced conditions}

Modeling an unbalanced dynamical power is a very difficult task. Indeed, lots of the methods present in the literature are not able to handle the unbalance. Well known methods are only able to handle single phase systems where unbalance is not possible or balanced multiphase systems. Applied to systems with unbalance, their performances are therefore necessarily moderate. It is therefore difficult to compare their performances to the one obtained with method introduced in the previous section which will necessarily be better.

\subsection{Context}

The ability of the new approach in estimating the fundamental frequency and the symmetrical components of power systems is investigated and evaluated. A set of simulation tests has been performed under different operating conditions. In the following tests, a power system composed of three phases with a fundamental frequency $f=50 \mathrm{~Hz}$ is used. A sampling time $T_{s}$ of $0.5 \mathrm{~ms}$ is chosen. 
A typical unbalanced power system is considered in this test:

$$
\left\{\begin{aligned}
v_{a}(k)=V_{+} \sin \left(\omega k T_{s}+\phi_{+}\right)+V_{-} \sin \left(\omega k T_{s}+\phi_{-}\right)+V_{o} \sin \left(\omega k T_{s}+\phi_{o}\right) \\
v_{b}(k)=V_{+} \sin \left(\omega k T_{s}+\phi_{+}+2 \pi / 3\right)+V_{-} \sin \left(\omega k T_{s}+\phi_{-}-2 \pi / 3\right) \\
\quad+V_{o} \sin \left(\omega k T_{s}+\phi_{o}\right) \\
v_{c}(k)=V_{+} \sin \left(\omega k T_{s}+\phi_{+}-2 \pi / 3\right)+V_{-} \sin \left(\omega k T_{s}+\phi_{-}+2 \pi / 3\right) \\
+V_{o} \sin \left(\omega k T_{s}+\phi_{o}\right)
\end{aligned}\right.
$$

The following numerical values are chosen in our simulations: $V_{+}=1, V_{-}=$ $0.2, V_{o}=0.1, \phi_{+}=\pi / 3, \phi_{-}=\pi / 6$ and $\phi_{o}=0$. The three phase signals of this system are represented by Fig. 2. The objective here consists in using an EKF with the proposed state-space in order to estimate the fundamental frequency at each iteration.

The power system that has been chosen and used in the tests is a simple one in order to be easily reproduced. Voltages with an amplitude of $1 \mathrm{~V}$ gives a good reference for comparing the results (per unit) and a nominal fundamental frequency of $50 \mathrm{~Hz}$ has been chosen, but the approach presents the same performance with any other values. Furthermore, the unbalance which is introduce is typical of the behavior of a power system in real-world applications.

\subsection{Results}

The proposed method is used to estimate the fundamental frequency of a power system under different conditions. The results are compared to the one obtained with the well-known method described in [7]. For each of these tests,
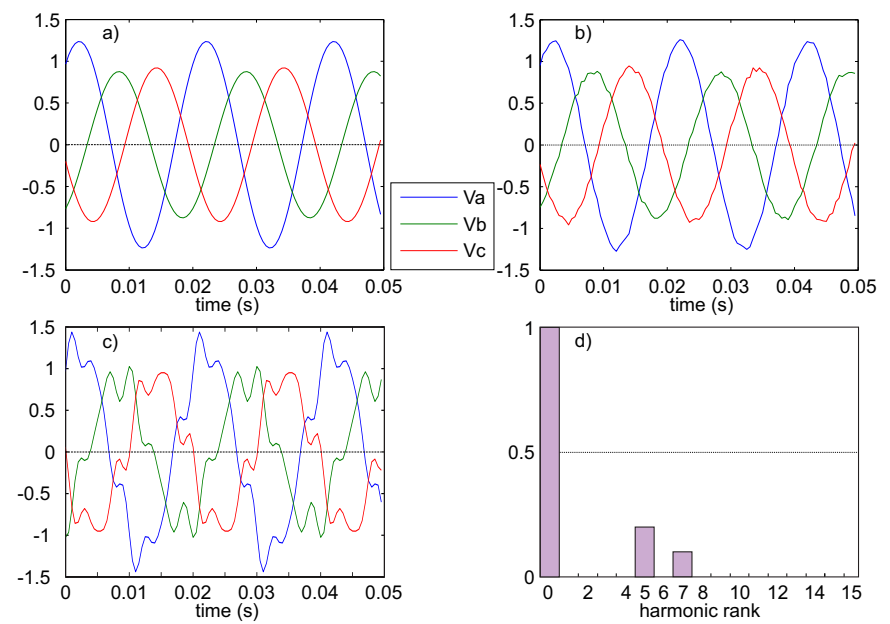

Fig. 2 The signals of the unbalanced three-phase system defined by Eq. 31, a) without noise, b) with a $30 \mathrm{~dB}$ noise, c) with higher-order harmonics of rank 5 and 7 , d) histogram corresponding to the signal with higher-order harmonics of rank 5 and 7 


\begin{tabular}{cccc}
\hline & time to reach the target & MSE at & max. error at \\
methods & freq. with $+/-0.1 \mathrm{~Hz}(\mathrm{~s})$ & steady-state $(\mathrm{Hz})$ & $\begin{array}{c}\text { steady-state }(\mathrm{Hz}) \\
\text { sthod of }[7]\end{array}$ \\
metho & 0.0732 & $2.2410^{-5}$ & $1.510^{-2}$ \\
proposed method & 0.0251 & $410^{-7}$ & $10^{-3}$ \\
\hline
\end{tabular}

Table 1 Performance of the proposed method compared to the method of [7] in estimating the frequency of an unbalanced system

\begin{tabular}{cccc}
\hline & time to reach the target & MSE at & max. error at \\
methods & freq. with $+/-0.1 \mathrm{~Hz}(\mathrm{~s})$ & steady-state $(\mathrm{Hz})$ & $\begin{array}{c}\text { steady-state }(\mathrm{Hz}) \\
\text { stea }\end{array}$ \\
\hline method of $[7]$ & 0.0637 & $310^{-5}$ & $1.610^{-2}$ \\
proposed method & 0.0245 & $1.710^{-6}$ & $310^{-3}$ \\
\hline
\end{tabular}

Table 2 Performance of the proposed method compared to the method of [7] in estimating the frequency of an unbalanced system with an additional $30 \mathrm{~dB}$ noise

\begin{tabular}{cccc}
\hline & time to reach the target & MSE at & max. error at \\
methods & freq. with $+/-0.1 \mathrm{~Hz}(\mathrm{~s})$ & steady-state $(\mathrm{Hz})$ & $\begin{array}{c}\text { steady-state }(\mathrm{Hz}) \\
\text { stea }\end{array}$ \\
\hline Method of [7] & 0.0927 & $5.610^{-5}$ & $2.410^{-2}$ \\
proposed method & 0.0409 & $1.710^{-6}$ & $510^{-3}$ \\
\hline
\end{tabular}

Table 3 Performance of the proposed method compared to the method of [7] in estimating the frequency of an unbalanced system disturbed by harmonics

the new state-space model is based on three state variables including the positive and negative components while the model of [7] uses only 2 states, either the positive or negative component. The initial conditions of the EKF are the first estimation of the frequency $\hat{f}(0)=45 \mathrm{~Hz}$ and $1.2 I$ for the error covariance matrix of the state estimate, $I$ is the identity matrix. The initial values of EKF's parameters are chosen in order to lead to a very simple configuration. These values are therefore deliberately not optimized. Obviously, optimizing this values will necessarily lead to faster convergence for estimating the fundamental frequency and the symmetrical components.

The proposed method and the method of [7] are both applied to estimate the fundamental frequency of the system given by Eq. 31. At steady-state, the estimated frequency given by [7] oscillates around the true value. The estimation provided by the proposed method does not oscillate. The efficiency of both methods in estimating the fundamental frequency is shown by Tab. 1. It can be seen that the estimated frequency obtained with the proposed method is highly accurate (the MSE is about $10^{-7} \mathrm{~Hz}$ ). As a comparison, the MSE with the method of [7] is 50 times bigger.

The immunity of the methods to noise is investigated by adding a zeromean white Gaussian noise with a Signal-to-Noise Ratio (SNR) of $30 \mathrm{~dB}$ to the three signals of Eq. 31. Tab. 2 shows a MSE in the range of $10^{-6} \mathrm{~Hz}$ for the estimated frequency with the proposed method. At the same time, the corresponding MSE for the method of [7] is in the range of $10^{-5} \mathrm{~Hz}$. These results prove that the proposed method is able to estimate the fundamental frequency quickly and accurately even under noisy conditions. 


\begin{tabular}{c|c|c|c}
\hline case & a) & b) & c) \\
\hline freq. step amplitude (Hz) & 0.00001 & 0.0001 & 0.001 \\
\hline peak value $(\mathrm{Hz})$ & 50.00024534 & 50.00245443 & 50.02454623 \\
\hline PO (\%) & 2354.34 & 2354.43 & 2354.62 \\
\hline first peak time $(\mathrm{ms}) 6.5$ & 6.5 & 6.5 & \\
\hline settling time to 5\% (ms) & 18 & 34 & 48 \\
\hline steady-state error $(\mathrm{Hz})$ & $3.16910^{-12}$ & $3.13210^{-11}$ & $3.12710^{-10}$
\end{tabular}

Table 4 Performance of the proposed state-space method in tracking fundamental frequency steps

The presence of harmonic terms influences the fundamental frequency estimation. This is evaluated for the two methods. Each phase signal of the system in Eq. 31 are therefore disturbed by adding higher order harmonics of rank 5 and 7 , respectively with amplitudes of $\frac{V_{+}}{5}$ and $\frac{V_{+}}{10}$. Results are presented in Tab. 3. It can be seen than the proposed method is robust against harmonic pollution. This is not the case of the other method.

Fig. 3 shows a good comparison of the proposed method with the previous one [7] in estimating frequency with noise and harmonics. The model proposed in [7] is flawed for modeling unbalanced systems. Thus, estimating the frequency is even more challenging for it under severe conditions, i.e., with the presence of noise and harmonics. As a consequence, the estimation is achieved with a low convergence rate, a constant steady-state error and oscillations. On the other hand, the state-space proposed in Eq. 11 models power systems with their unbalance properties. Therefore, this model is efficient in estimating the frequency in an EKF scheme and is robust against noise and higher-order harmonics. Additional tests have demonstrated that proposed the state-space is capable of estimating the frequency under balanced conditions.

To test the speed and convergence of the proposed technique at nominal frequency conditions (the fundamental frequency is $f_{0}=50 \mathrm{~Hz}$ but it can be any other numerical value), a signal of known harmonic contents is taken for fundamental frequency estimation where frequency steps occur under unbalanced conditions. In this test, fundamental frequency steps appear with different amplitudes, i.e., 0.001, 0.0001 and $0.00001 \mathrm{~Hz}$. The fundamental frequency is estimated by using the proposed state-space model and the EKF algorithm. The fundamental frequency step appears at instant $0.25 \mathrm{~s}$, in other words at iteration 500 .

The simulation results reveal that the proposed state-space method takes few iterations to reach the convergent value of new value of the fundamental frequency. Results are represented for each case in Table 4 which indicates the Percent Overshoot (PO), the peak value, the first peak time, the settling time to within $5 \%$ of the final value and the steady-state error. If the peak values are high in percent, frequency steps of $0.001 \mathrm{~Hz}$ are very severe cases and reasonably never happen in real installations, and a frequency step of 0.1 $10^{-3} \mathrm{~Hz}$ leads to a overshoot of $2.45410^{-4} \mathrm{~Hz}$. Furthermore, the fundamental frequency estimated by the proposed state-space model and the EKF converges rapidly the real value of the frequency. In all cases, the peak value appears only 

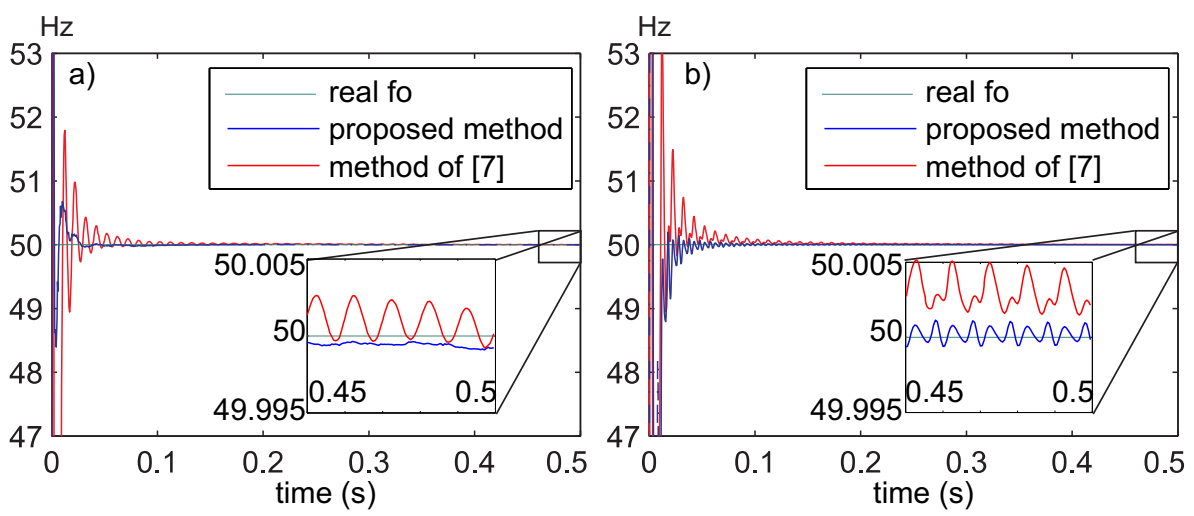

Fig. 3 Estimated frequencies by the two methods, the proposed one and, a) with a $30 \mathrm{~dB}$ noise, b) with harmonics of rank 5 and 7

12 iterations after the fundamental frequency step, i.e., in less than $6.5 \mathrm{~ms}$. Table 4 also shows the accuracies of the proposed estimator: At steady-state, the error is almost less than $3.210^{-10} \mathrm{~Hz}$.

All these results demonstrates that the proposed state-space method is an accurate frequency estimator even in time-varying environments and for fundamental frequency variations.

\section{Symmetrical components estimation under unbalanced conditions}

The proposed state-space model is used in an EKF scheme to identify at each instant the symmetrical components of the unbalanced power systems given by Eq. 31 with the following numerical values: $V_{+}=1, V_{-}=0.4, V_{o}=0.1$ and $\phi_{+}=\pi / 3, \phi_{-}=\pi / 6, \phi_{o}=0$. A zero-mean, white Gaussian noise of $30 \mathrm{~dB}$ is added to each of the signals. The initial values of state variables $q_{1}(k), q_{2}(k)$ and $q_{3}(k)$ are respectively chosen as $0.9891+0.1471 i$ (this value corresponds to the fundamental frequency at $47 \mathrm{~Hz}$, i.e., a random value close to the supposed fundamental frequency), $1.2-0.5 i$ and 0 . The error covariance matrix of the state estimate is initialized at $1.2 \mathbf{I}$.

\subsection{Steady-state results}

A first test evaluates the performance of the proposed method at steady-state and under disturbed conditions, i.e., with noise and harmonic distortions. Table 5 and Table 6 evaluate the mean values, the MSE and the maximum errors of the estimated amplitudes at steady-state and respectively with a 30DB noise and harmonic distortions (higher-order harmonics of rank 5 and 7, respectively with amplitudes of $\frac{I_{+}}{10}$ and $\left.\frac{I_{+}}{15}\right)$. The mean values indicate the biases from the true values. It can be explained as the result of a poor initialization of the 


\begin{tabular}{cccc}
\hline Amplitudes & $\begin{array}{c}\text { mean at } \\
\text { steady-state } \\
(\mathrm{A})\end{array}$ & $\begin{array}{c}\text { MSE at } \\
\text { steady-state } \\
(\mathrm{A})\end{array}$ & $\begin{array}{c}\text { Error max. } \\
\text { at steady-state } \\
(\mathrm{A})\end{array}$ \\
\hline Positive component & 0.9988 & $2.862710^{-6}$ & $4.610^{-3}$ \\
Negative component & 0.4011 & $1.735910^{-6}$ & $3.210^{-3}$ \\
\hline
\end{tabular}

Table 5 Performance of the new method for estimating the amplitudes of the positive and negative components of three phase signals disturbed by noise of $30 \mathrm{~dB}$

\begin{tabular}{cccc}
\hline Amplitudes & $\begin{array}{c}\text { mean at } \\
\text { steady-state } \\
(\mathrm{A})\end{array}$ & $\begin{array}{c}\text { MSE at } \\
\text { steady-state } \\
(\mathrm{A})\end{array}$ & $\begin{array}{c}\text { Error max. } \\
\text { at steady-state } \\
(\mathrm{A})\end{array}$ \\
\hline Positive component & 0.9981 & $5.234910^{-6}$ & $8.310^{-3}$ \\
Negative component & 0.4008 & $9.270610^{-7}$ & $3.110^{-3}$ \\
\hline
\end{tabular}

Table 6 Performance of the new method for estimating the amplitudes of positive and negative components of three phase signals disturbed by harmonics of rank 5 and 7

EKF. However, the MSE is in the range of $10^{-6}$ and the maximum error is in the range of $10^{-3}$. This demonstrates that the deviation of the estimated amplitudes from the true ones is small and that the method provides high accuracy in estimating the amplitudes of the symmetrical components. The proposed method could be enhanced to track every individual high-order harmonic component.

\subsection{Dynamic behavior}

A second test is proposed in order to evaluate the dynamics of the proposed method, i.e., its robustness against time-varying load changes. We propose to switch suddenly from a balanced load to an unbalanced one. The following three-phase balanced system is considered to start the test:

$$
\left\{\begin{array}{l}
v_{a}(k)=\sin \left(\omega k T_{s}\right) \\
v_{b}(k)=\sin \left(\omega k T_{s}-2 \pi / 3\right) \\
v_{c}(k)=\sin \left(\omega k T_{s}+2 \pi / 3\right)
\end{array}\right.
$$

At instant $0.075 \mathrm{~s}$ (at iteration $k=150$ ), it becomes an unbalanced system defined by Eq. 31 with $V_{+}=0.8, V_{-}=0.2, V_{o}=0.1, \phi_{+}=\frac{\pi}{3}, \phi_{-}=\frac{\pi}{2}$ and $\phi_{o}=0$. The proposed method is used to identify the symmetrical components of the changing system at each instant.

Results are presented by Fig. 4 and it can be seen that during at the first period of time, i.e., $k<150$, the estimated negative component is zero while the estimated positive component has an amplitude of $1 \mathrm{~V}$ and an phase angle of $0 \mathrm{rad}$ at steady-state. After the failure appears at iteration $k=150$ and after a short transient, the estimated amplitudes of the positive and negative components converge respectively to $0.80 \mathrm{~V}$ and $0.20 \mathrm{~V}$. At the same time, the estimated phase angles converge to 1.047 and 1.571 respectively for the positive and negative components. Referring to Eq. 31 and Eq. 32, these values are all the true values of the power system's parameters. 

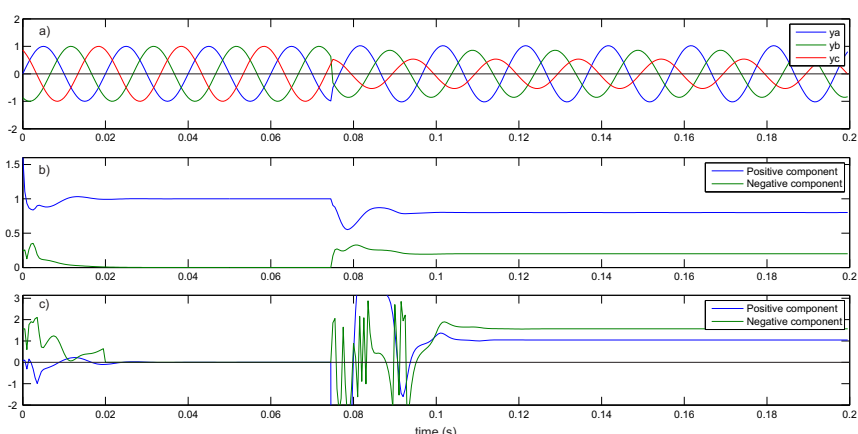

Fig. 4 Identification of the symmetrical components, a) the unbalanced three-phase signals, b) estimated amplitudes of the positive and negative components, and c) phase angles of the positive and negative components
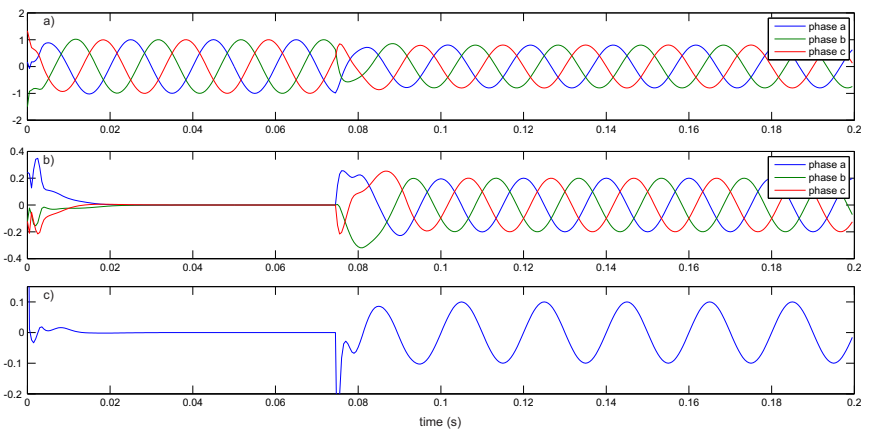

Fig. 5 Reconstruction of the system's symmetrical components, a) recovered positive components, b) recovered negative component, and c) recovered zero component

The evolution of the positive and negative components are shown by Fig. 5 . This figure also shows the evolution of the three currents reconstructed from the symmetrical components. The results show that after a time shorter than one cycle, the estimated currents are very close to the real values. The evolution of the symmetrical components converted in the $\alpha \beta$ reference frame are shown by Fig. 6. It can be seen from this figure that at the beginning, the reconstructed system is balanced. After the failure appears, the negative and zero component become significant and the positive component decreases at the same time. This characterizes the behavior of the true power system.

Finally, the estimation performance have been evaluated with a continuously changing fundamental frequency of the power system. From the instant $0.2 \mathrm{~s}$, the fundamental frequency speed is $-2 \mathrm{~Hz} / \mathrm{s}$. This is illustrated by Fig. 7 . The evolution of the fundamental frequency is shown with also the frequency error and the symmetrical component errors (positive and negative) for the three phases. The frequency error is less than $2.2510^{-3} \mathrm{~Hz}$ and the error in estimating the symmetrical is less than $1.510^{-5}$. These are acceptable ranges in power systems applications. 


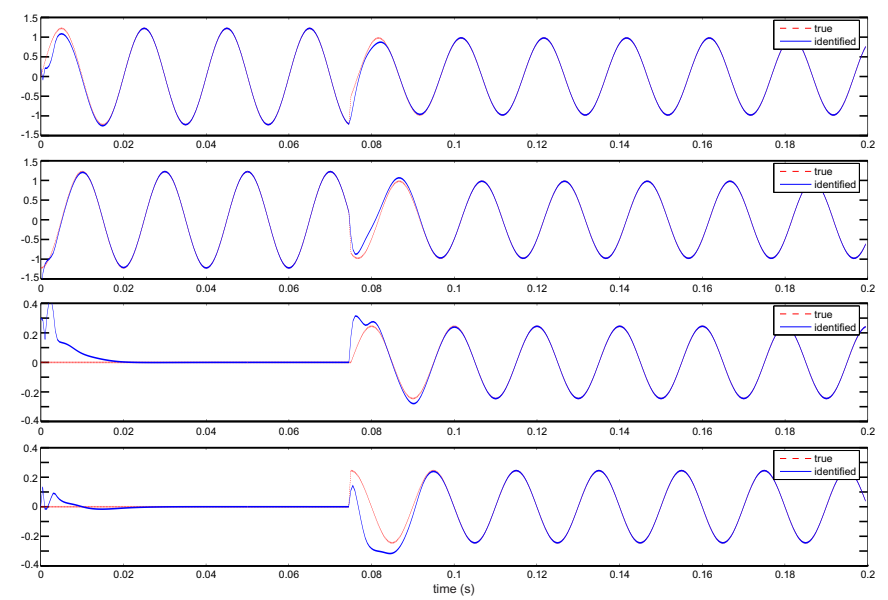

Fig. 6 Identified voltages compared to the true ones, a) $v_{\alpha}^{+}$, b) $v_{\beta}^{+}$, c) $v_{\alpha}^{-}$and d) $v_{\beta}^{-}$

\section{Conclusion}

A new state-space representation has been developed in order to estimate the parameters of three phase power systems. The new state-space model is combined with an extended Kalman filter and allows to iteratively estimate the fundamental frequency and the symmetrical components of a dynamic three-phase power system. The state-space is composed of the positive and negative components of the three-phase system and by the variation of the phase angle of them between two consecutive iterations. As a consequence, the state-space is not only able to model balanced three-phase power systems but also unbalanced three-phase systems. Numerical simulations were performed to evaluate the performance of the new approach in different conditions: Unbalanced systems with the presence of harmonics or with noise, system changes from balance to unbalance, slow and abrupt frequency changes. With this new approach, the fundamental frequency and the symmetrical components of a three-phase power system are estimated with high accuracy under disturbed conditions. Simulation results show that the proposed state-space associated to the extended Kalman filter is efficient. The symmetrical components of a time-varying power system can thus be reconstructed under various severe unbalanced conditions. Phase angles of the symmetrical components are sensitive to disturbances such as noises and harmonics. On the contrary, their amplitudes reflects the unbalance and must be considered in power quality control strategies. The proposed method works well for unbalanced power systems without any knowledge. We considered power distribution systems but the method can also be applied to any electric drive or generator. We considered the three-phase voltages but the proposed development can be generalized to any three-phase signals, i.e., currents. The proposed approach is fully adaptive and can therefore react very quickly to any power system changes in real-time. 

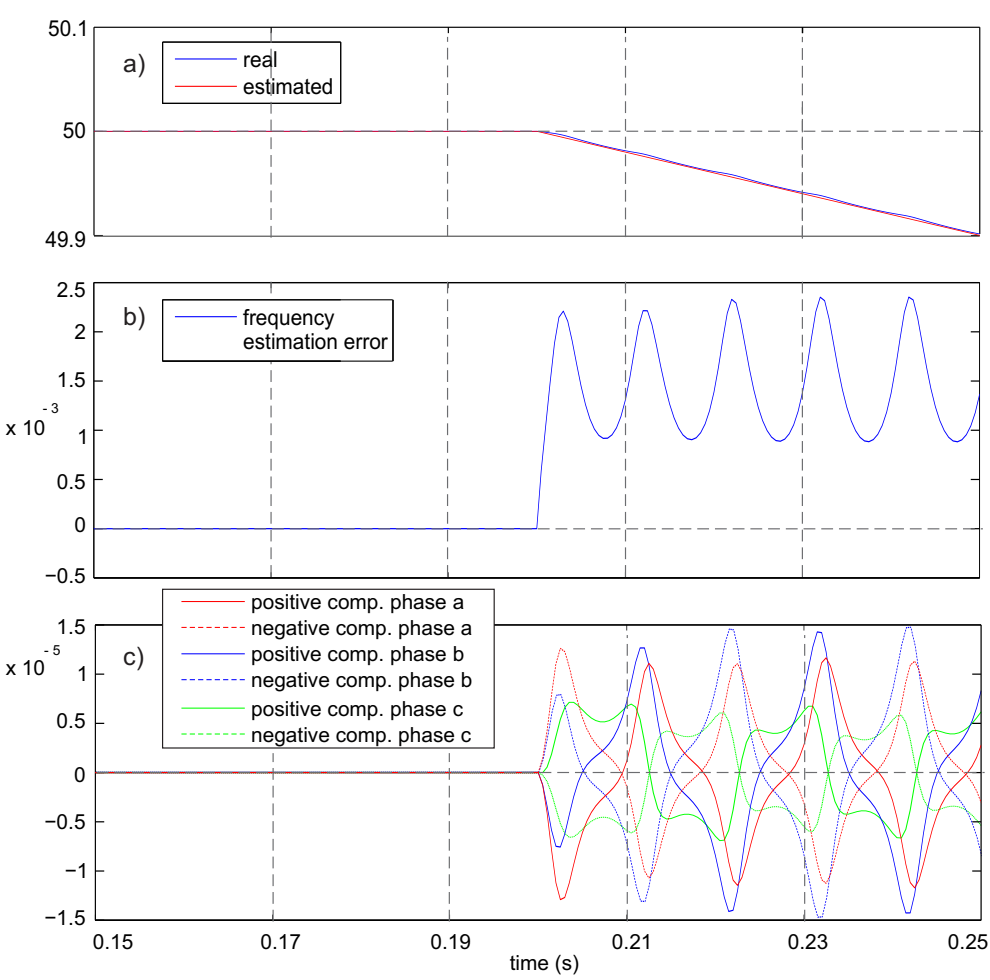

Fig. 7 Performance in estimating the frequency and the symmetrical components with a changing fundamental frequency, a) real and estimated frequency $(\mathrm{Hz}), \mathrm{b})$ frequency error, and c) symmetrical components errors

\section{References}

1. Akagi, H., Watanabe, E.H., Aredes, M.: Instantaneous Power Theory and Applications to Power Conditioning. Wiley (2007)

2. Arrillaga, J., Smith, B.C., Watson, N.R., Wood, A.R.: Power System Harmonic Analysis. John Wiley and Sons (1997)

3. Baggini, A.: Handbook of Power Quality. John Wiley and Sons (2008). DOI $10.1002 / 9780470754245$

4. Blackburn, J.L.: Symmetrical Components for Power Systems Engineering. CRC Press (1993)

5. Boashash, B., Lovell, B., Kootsookos, P.: Time-frequency signal analysis and instantaneous frequency estimation: methodology, relationships and implementations. In: IEEE International Symposium on Circuits and Systems (ISCAS 1989), pp. 1237-1242 (1989)

6. Chan, K., So, H.: Accurate frequency estimation for real harmonic sinusoids. IEEE Signal Processing Letters 11(7), 609-612 (2004)

7. Dash, P., Pradhan, A., Panda, G.: Frequency estimation of distorted power system signals using extended complex kalman filter. IEEE Transactions on Power Delivery 14(3), $761-766$ (1999)

8. Dash, P., Swain, D., Routray, A., Liew, A.: An adaptive neural network approach for the estimation of power system frequency. Electric Power Systems Research 41(3), 203-210 (1997)

9. De La Rosa, F.C.: Harmonics, Power Systems, and Smart Grids, 2 edn. CRC Press (2015) 
10. Dugan, R.C., McGranaghan, M.F., Santoso, S., Beaty, H.W.: Electrical Power Systems Quality, 3 edn. McGraw-Hill Professional (2012)

11. Fu, H., Vong, C., Wong, P., Yang, Z.: Fast detection of impact location using kernel extreme learning machine. Neural Computing and Applications 27(1), 121-130 (2016)

12. Gonen, T.: Electric Power Distribution Engineering. CRC Press (2008)

13. Halbwachs, D., Wira, P., Merckle, J.: Adaline-based approaches for time-varying frequency estimation in power systems. In: 2nd IFAC International Conference on Intelligent Control Systems and Signal Processing (ICONS 2009) (2009)

14. Haykin, S.S.: Adaptive Filter Theory, 3rd edn. Prentice Hall Information and System Sciences Series. Prentice Hall (1996)

15. Huang, C.H., Yunlin, Lee, C.H., Shih, K.J., Wang, Y.J.: Frequency estimation of distorted power system signals using robust extended complex kalman filter. In: International Conference on Intelligent Systems Applications to Power Systems (ISAP 2007). Niigata, Japan (2007)

16. von Jouanne, A., Banerjee, B.: Assessment of voltage unbalance. IEEE Transactions on Power Delivery 16(4), 782-790 (2001)

17. Kalhor, A.: Potential of evolving ar and arx models in signal recovering. Evolving Systems 7(1), 61-72 (2016). DOI 10.1007/s12530-014-9114-0. URL http://dx.doi.org/10.1007/s12530-014-9114-0

18. Kanieski, J.M., Cardoso, R., Pinheiro, H., Gr, H.A., x00Fc, ndling: Kalman filter-based control system for power quality conditioning devices. IEEE Transactions on Industrial Electronics 60(11), 5214-5227 (2013). DOI 10.1109/TIE.2012.2226412

19. Lewis, F.L., Xie, L., Popa, D.: Optimal and Robust Estimation: With an Introduction to Stochastic Control Theory, 2 edn. CRC Press (2007)

20. Liccardo, F., Marino, P., Raimondo, G.: Robust and fast three-phase pll tracking system. IEEE Transactions on Industrial Electronics 58(1), 221-231 (2011). DOI 10.1109/TIE.2010.2044735

21. Mendon, T.R.F., x00E, Pinto, M.F., Duque, C.A.: Least squares optimization of zero crossing technique for frequency estimation of power system grid distorted sinusoidal signals. In: Industry Applications (INDUSCON), 2014 11th IEEE/IAS International Conference on, pp. 1-6 (2014). DOI 10.1109/INDUSCON.2014.7059443

22. Ould Abdeslam, D., Wira, P., Merckle, J., Flieller, D.: A unified artificial neural network architecture for active power filters. Electric Power Systems Research 54(1), 61-76 (2007)

23. Phan, A.T., Hermann, G., Wira, P.: Online frequency estimation in power systems: A comparative study of adaptive methods. In: 40th Annual Conference of the IEEE Industrial Electronics Society (IECON 2014), pp. 4352-4357 (2014)

24. Phan, A.T., Hermann, G., Wira, P.: Kalman filtering with a new state-space model for three-phase systems: Application to the identification of symmetrical components. In: IEEE Conference on Evolving and Adaptive Intelligent Systems (EAIS 2015) (2015)

25. Phan, A.T., Hermann, G., Wira, P.: A new state-space for unbalanced three-phase systems: Application to fundamental frequency tracking with kalman filtering. In: 18th IEEE Mediterranean Electrotechnical Conference (2016)

26. Pradhan, A., Routray, A., Basak, A.: Power system frequency estimation using least mean square technique. IEEE Transactions on Power Delivery 20(3), 1812-1816 (2005)

27. Reza, M., Ciobotaru, M., Agelidis, V.: Power quality analysis using piecewise adaptive prony's method. In: 2012 IEEE International Conference on Industrial Technology (ICIT). Athens, Greek (2012)

28. Rubio, J.d.J.: Adaptive least square control in discrete time of robotic arms. Soft Computing 19(12), 3665-3676 (2015)

29. Rubio, J.d.J.: Least square neural network model of the crude oil blending process. Neural Networks 78, 88-96 (2016). DOI http://dx.doi.org/10.1016/j.neunet.2016.02.006

30. Rubio, J.d.J., Ochoa, G., Meda, J.A., Rangel, V.I., J., P.: Acquisition system and analytic fuzzy model of a manufactured wind turbine. IEEE Latin America Transactions 13(12), 3879-3884 (2015)

31. Seyedi, H., Sanaye-Pasand, M.: A new time-domain based power system frequency estimation algorithm. European Transactions on Electrical Power 22(4), 433-448 (2012). DOI 10.1002/etep.572. URL http://dx.doi.org/10.1002/etep.572 
32. Shayeghi, H., Shayanfar, H.A., Jalili, A.: Load frequency control strategies: A state-ofthe-art survey for the researcher. Energy Conversion and Management 50(2), 344-353 (2009)

33. Shinnaka, S.: A robust single-phase pll system with stable and fast tracking. IEEE Transactions on Industry Applications 44(2), 624-633 (2008). DOI 10.1109/TIA.2008.916750

34. Toubakh, H., Sayed-Mouchaweh, M.: Hybrid dynamic data-driven approach for driftlike fault detection in wind turbines. Evolving Systems 6(2), 115-129 (2015). DOI $10.1007 / \mathrm{s} 12530-014-9119-8$

35. Yang, J.Z., Liu, C.W.: A precise calculation of power system frequency. IEEE Transactions on Power Delivery 16(3), 361-366 (2001) 